\title{
Sonografiekurse nach den Richtlinien der ÖGUM
}

Detaillierte Infos im Kurs-Kalender unter : www.oegum.at/ausbildung/kurskalender. html

\section{Abdomen}

- 01.10.-03.10.2014 Linz: Grundkurs Abdomen 28.01.-30.01.2015 Linz: Aufbaukurs Abdomen Leitung \& Auskunft: OA Dr. G. Schneider; kornelia.soellinger@elisabethinen.or.at

15.10.-16.10.2014 Baden: Grundkurs Abdomen

Leitung \& Auskunft: Prof. Dr. N. Gritzmann; norbert.gritzmann@gmail.com; Tel. 0043662840464

- 11.09.-13.09.2014 Hohenems: Grundkurs Abdomensonografie

02.10.-04.10.2014 Hohenems: Aufbau-/Re-

fresherkurs Abdomensonografie

Leitung \& Auskunft: OA Dr. Gehmacher; sekretariat.interne@lkhh.at

- 19.09.-20.09.2014 Salzburg: Gefäßbasiskurs \& Small-Parts Kurs

19.11.-21.11.2014 Salzburg: Grundkurs Abdomen
Leitung \& Auskunft: OA Dr. Hollerweger; kontakt@bhb-sonokurse.at

- 06.10.-07.10.2014 Graz: Abdominelle Sonografie Grundkurs (ÖGUM) mit E-Learning Leitung \& Auskunft: Prim.Dr. Simader; fortbildung@aekstmk.or.at

Bewegungsapparat / Nervensystem

- 25.09.-27.09.2014 Wien: 5th Interdisciplinary Muscoloskeletal Ultrasound Course Leitung \& Auskunft: PD Dr. Schüller-Weidekamm; us-course-vienna@meduniwien.ac.at

- 15.11.-16.11.2014 Innsbruck: Sonografie des peripheren Nervensystems Leitung \& Auskunft: Doz.Dr. Gruber; karoline. volderauer@i-med.ac.at

Gynäkologie/Geburtshilfe

- 19.09.-20.09.2014 Graz: Ultraschall konkret/ plus 
Leitung \& Auskunft: Univ.Prof. Dr. M. Häusler; martin.haeusler@medunigraz.at

\section{Kopf/Hals/Gefäße}

- 17.10.-18.10.2014 Baden: Der Halskurs (Duplexsonografie Halsgefäße, Schilddrüse, Halsweichteile)

Leitung \& Auskunft: Prof. Dr. N. Gritzmann; norbert.gritzmann@gmail.com; Tel. $0043662-$ 840464

- 19.09.-20.09.2014 Salzburg: Gefäßbasiskurs \& Small-Parts Kurs

Leitung \& Auskunft: OA Dr. Hollerweger; kontakt@bhb-sonokurse.at

- 06.10.2014 Graz: Schilddrüsensonografie Leitung \& Auskunft: Dr Buchinger; fortbildung@aekstmk.or.at

- 07.11.-08.11.2014 Hohenems: Klinische Endokrinologie und Schilddrüsenultraschallkurs I Leitung \& Auskunft: Dr. Höfle/Gessle
- 15.11.-16.11.2014 Innsbruck: Doppler- und Duplexsonografie der hirnversorgenden Arterien

Leitung \& Auskunft: Dr. Schmidauer; christoph. schmidauer@uki.at

\section{Pädiatrie}

- 13.11.-16.11.2014 Eisenerz: Aufbaukurs 23. Steirisches Seminar „Pädiatrische Ultraschalldiagnostik“ Leitung \& Auskunft: OA Dr. G. Schweintzger; gerolf.schweintzger@lkh-leoben.at

- 19.09.-20.09.2014 Stolzalpe: Intensivkurs Sonografie der Säuglingshüfte nach Graf

- 05.12.-06.12.2014 Stolzalpe: Intensivkurs Sonografie der Säuglingshüfte nach Graf Leitung \& Auskunft: Doz.Dr. Tschauner; christine.puff@LKH-Stolzalpe.at 